\title{
Passive Acoustic Knock Tracking for Interactive Windows
}

\author{
Joseph A. Paradiso, Che King Leo, Nisha Checka, Kaijen Hsiao \\ Responsive Environments Group \\ MIT Media Laboratory \\ 1 Cambridge Center, 5FL \\ Cambridge, MA 02142 USA \\ \{joep,cheking,nchecka,kjhsiao\}@media.mit.edu
}

\begin{abstract}
We describe a novel interface that locates and characterizes knocks and taps atop a large glass window. Our current setup uses four contact piezoelectric pickups located near the sheet's corners to record the acoustic wavefront coming from the knocks. A digital signal processor extracts relevant characteristics from these signals, such as amplitudes, frequency components, and differential timings, which are used to estimate the location of the hit and provide other parameters, including the rough accuracy of this estimate, the nature of each hit (e.g., knuckle knock, metal tap, or fist bang), and the strike intensity. This system requires only simple hardware, needs no special adaptation of the glass pane, and allows all transducers to be mounted on the inner surface, hence it is quite easy to deploy as a retrofit to existing windows. This opens many applications, such as an interactive storefront, with projected content controlled by knocks on the display window.
\end{abstract}

\section{Keywords}

touch screen, interactive surface, acoustic tracking

\section{INTRODUCTION}

Glass is now a very common construction material, often used as clear walls for room dividers or large windows enclosing urban buildings. The techniques described in this paper enable these surfaces to become interactive. For example, information displayed on a projection or monitor on the inside of the glass can be navigated by knocking appropriately on the outside. A straightforward application of this niche is an interactive storefront, where passers-by can browse information on the store's merchandise or explore special offers by knocking appropriately.

In general, most technologies used in conventional touch screens [1] don't scale gracefully to large displays and/or require hardware to be mounted on the outside surface of the display, leading to limited longevity for outdoor or public installations. This is certainly true of common resistive techniques and IR interrupter approaches.

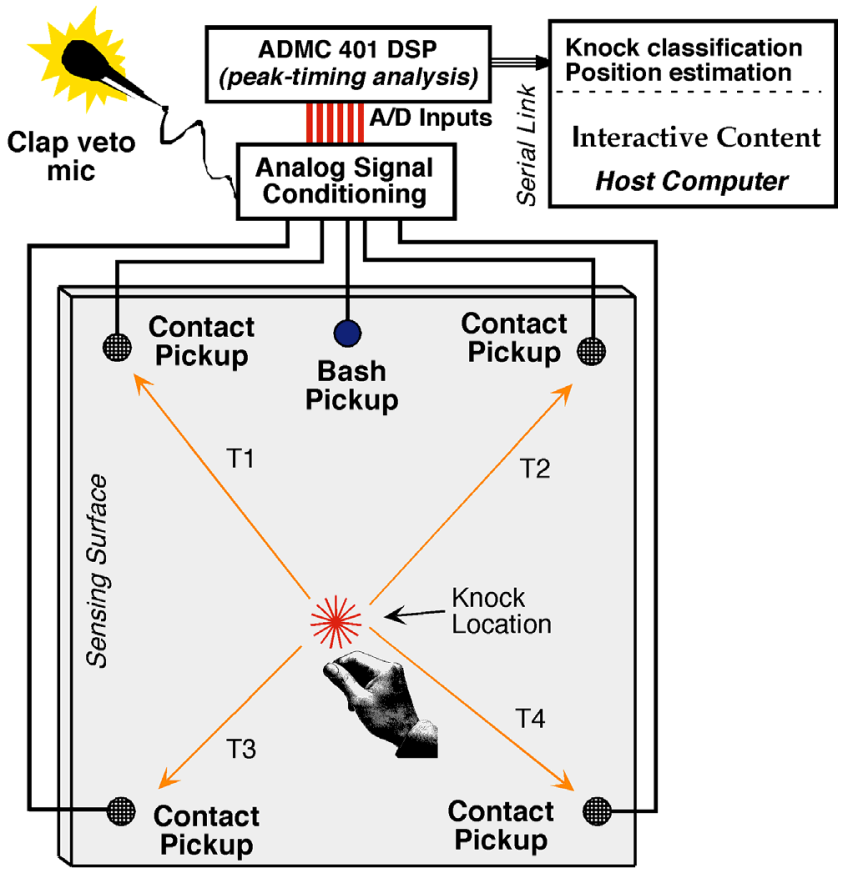

Figure 1: Hardware configuration for knock tracker system

Capacitive and active acoustic techniques require the glass to be patterned with transparent electrodes or waveguides, which can be expensive and problematic over large areas.

Other techniques, such as video tracking [2] have been used to make large screens interactive. Although they are steadily improving, such vision-based approaches can be slow and are often sensitive to image clutter, target reflectance, and changes in background lighting. Laser rangefinding has also been used to scan the surface of large displays [3] and track hands, but the potentially expensive laser scanner must be mounted outside the window, leading to reliability difficulties for outdoor operation.

\section{PASSIVE ACOUSTIC IMPACT TRACKING}

Figure 1 shows a block diagram of our system. An array of four piezoelectric pickups, one on each corner of the glass, locates the position of a knock through the differences between times of the wavefront arrival at each sensor. The amplitude of the signals determines the intensity of the knock, and the frequency determines whether the knock came from a "soft" knuckle tap or "hard" metallic tap. An additional electrodynamic transducer mounted on the glass can easily discriminate low-frequency "fist bashes" from 
other types of knocks, and a "Clap veto" microphone in the air nearby prevents loud, sharp, external sounds from creating false triggers in very noisy environments.

Although the original motivation for this system was to track the location of knocks on a virtual fishtank [4], the first implementation of this technique was realized in a collaboration of the lead author with Hiroshi Ishii's Tangible Media Group for the PingPong+ $(\mathrm{PP}+)$ augmented ping pong table [5]. Our current application required the development of more sophisticated techniques to time the signals, as the knock of a knuckle on glass is much less distinct than the impact of a ping-pong ball on wood. Similarly, the bending waves launched into the glass are highly dispersive, creating significant distortion in the received audio waveforms and resulting in propagation velocities that varied for different types of hits [3]. Crosscorrelation and heuristic peak-matching algorithms were developed [6] to solve these problems. Our resulting system runs sample-intensive operations on a low-cost embedded DSP (the ADMC401 from Analog Devices), which transfers a set of extracted wavefront features to a PC that runs a simple hyperbolic calibration and a tracking routine. The net latency of this system is low - coordinates are produced within $65 \mathrm{~ms}$, with the DSP algorithms dominating this delay. The accuracy of the knock position estimate varies with the kind of glass used and the type of hit. For a $1-\mathrm{cm}$ thick, $1 \times 1$ meter glass pane, we obtain position resolutions under $\sigma=3.5 \mathrm{~cm}$. For a $0.5-\mathrm{cm}$ thick, $1.5 \times 1.0$ meter glass pane, we see a better resolution; e.g., $\sigma=2.5 \mathrm{~cm}$. This is due to the difference in propagation characteristics - bending waves travel about $700 \mathrm{~m} / \mathrm{s}$ for knuckle and $1700 \mathrm{~m} / \mathrm{s}$ for metal taps in $1 \mathrm{~cm}$ glass; speeds are roughly $20-30 \%$ slower in $0.5 \mathrm{~cm}$ glass.

\section{APPLICATIONS}

Starting with an initial diagnostic that projected a circle around the estimated knock location [3], we improved the system until we were able to run a knock-driven browser [6], where users could tap on various icons to navigate a through a database of group projects and demo videos. We then developed an installation called the "Responsive Window" [7] that is now running at the Ars Electronica Center in Linz, Austria. This is a knock-driven drawing program developed in collaboration with Ben Fry of the Media Lab's Aesthetics \& Computation Group. As indicated in Figure 2, the user can extrude and animate various 3D shapes as they tap and knock. Next we collaborated with video artist J.D. Beltran in "Telephone Story" [8], now running at the Kitchen gallery in Manhattan. Here users can select videos and view hidden images by knocking in the appropriate areas of a large display. We are currently installing the knock tracker system at a storefront near Rockefeller Center in Manhattan; even while the store is closed, pedestrians can navigate projected information by knocking at the window, lending a modern spin to the term "window browsing".

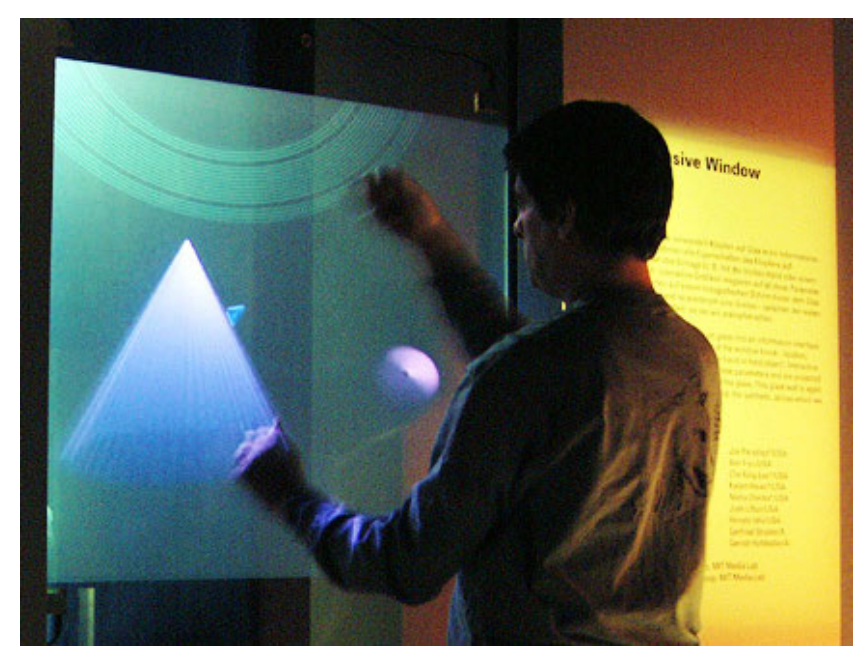

Figure 2: The Responsive Window in action

\section{CONCLUSIONS}

We have demonstrated a technique of easily retrofitting common windows for contact interactivity by measuring the position of a knock or tap, determining the type of impact, and estimating the impact intensity. We have installed this system in several public venues, most recently at a large storefront, where we can explore applications in interactive retail. Once users realize that they are invited to knock on the glass (a common pre-digital gesture), they have little difficulty and adapt well to the interaction.

\section{ACKNOWLEDGMENTS}

We acknowledge our aforementioned artistic collaborators, Steve Francis of Analog Devices for DSP help, and our colleagues Josh Lifton and Warit Wichakol for their work with early versions of this system. We thank our MIT Media Laboratory sponsors for supporting this project.

\section{REFERENCES}

1. Quinnell R.A., "Touchscreen technology improves and extends its options," EDN, vol.40, no.23, 9 Nov. 1995, pp.52, 54-6, 58, 60, 62, 64.

2. Rekimoto, J., Matsushita, N., "Perceptual Surfaces: Towards a Human and Object Sensitive Interactive Display. In Workshop on Perceptual User Interfaces (PUI-97), Banff, October 1997, pp. 30-32.

3. Paradiso, J.A., et al, "Sensor Systems for Interactive Surfaces," IBM Systems Journal, Vol. 39, No. 3\&4, October 2000, pp. 892-914.

4. Galyean, T., et al., "Virtual FishTank," SIGGRAPH 98, Conf. Abstracts and Applications, ACM Press 1998, p. 116.

5. Ishii, H., Wisneski, C., Orbanes, J., Chun, B., and Paradiso, J., "PingPongPlus: Design of an Athletic-Tangible Interface for Computer-Supported Cooperative Play," in Proceedings of CHI'99, pp. 394-401.

6. Checka, N., "A System for Tracking and Characterizing Acoustic Impacts on Large Interactive Surfaces," MS Thesis, MIT Department of EECS and MIT Media Lab, May 2001.

7. Paradiso, J., "Responsive Window," in Take Over, Proc. of 2001 Ars Electronica Festival, Springer Verlag,, pp. 261-263.

8. See: http://identity.media.mit.edu/telephonestory.html 